\title{
Análisis psicosocial del suicidio en personas jóvenes indígenas Bribris
}

\section{Psychosocial Analysis of Suicide in Young Bribris People}

\author{
Helga Arroyo Araya ${ }^{1}$ \\ Damián Gerardo Herrera González ${ }^{2}$
}

Fecha de recepción: 28 de setiembre de 2018 Fecha de aceptación: 10 de abril de 2019

\begin{abstract}
Resumen
Este artículo recupera el proceso reflexivo de construcción colectiva de saberes, realizado en conjunto con personas líderes, actores institucionales y personas jóvenes de las comunidades indígenas Bribris de "Sepecue, Shiroles, Suretka y Bambú", durante el periodo de agosto del 2015 a julio del 2017. Como principal conclusión se destaca que el suicidio es la expresión de un trauma psicosocial zanjado por prácticas sistemáticas de colonización (históricas, sociales, políticas, económicas y culturales), que han incidido en el debilitamiento de las identidades culturales y de la construcción subjetiva en el territorio indígena Bribri. Sin embargo, la investigación también permite concluir que el vínculo y el fortalecimiento de lo cultural, constituyen las vías de resistencia para afrontar esta problemática.

Palabras clave: suicidio, trauma psicosocial, vínculo, fortalecimiento cultural, resistencia indígena.
\end{abstract}

\begin{abstract}
This article portrays the experiences of a reflective process of a collective knowledge construction, carried out from August 2015 to July 2017 with leaders, institutional actors, and young people from the "Bribris" indigenous communities of "Sepecue", "Shiroles", "Suretka," and "Bambu". The main conclusion of this study was that suicide within this indigenous territory is the expression of a psychosocial trauma, which is the result of colonization systematic practices (historical, social, political, economic, and cultural practices) that have weakened the "Bribri" cultural identity and their indigenous subjective construction. Nevertheless, this research study also concludes that the cultural connection and reinforcement constitute the ways of resistance to face these issues.

KeyWords: suicide, psycho-social trauma, social bond, cultural strengthening and indigenous resistance.
\end{abstract}

1. Escuela de Psicología/Posgrado de Psicología, Universidad de Costa Rica, Costa Rica; Hospital Nacional Psiquiátrico, Costa Rica, helga.arroyo.araya@gmail.com

2. Escuela de Psicología/Centro de Investigaciones sobre Diversidad Cultural y Estudios Regionales, Universidad de Costa Rica, Costa Rica, damianhg@gmail.com

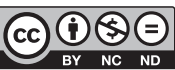


Por una Talamanca que lucha, resiste y mira a la vida.

\section{Introducción}

En el año 2014, en el cantón de Talamanca se estableció una declaratoria de emergencia cantonal por la problemática de suicidio en población joven: a raíz del reporte de 47 intentos de suicidio y 11 casos de suicidio consumados. Del número total de intentos, 22 de los casos correspondieron a personas del territorio indígena Bribri; mientras que, del total de suicidios consumados, 6 correspondían a personas habitantes de dicho territorio. Del total de casos de suicidio, el 90\% correspondían a personas menores de 35 años, y en tanto que el $27 \%$ de los casos totales correspondían a personas menores de edad (OIJ 2014; CCS 2014).

Considerando que, según los registros del Organismo de Investigación Judicial (OIJ) (2014), el número total de suicidios en Costa Rica durante el año 2014 fue de 302 personas. Si se considera que, según datos del INEC (2011), la población del cantón de Talamanca representa el 0,66\% de la población del país (Instituto Nacional de Estadística y Censo 2011), es posible sostener que una incidencia de 11 suicidios consumados en proporción a la estadística nacional, supone datos alarmantes.

En este marco, se sitúa la investigación «Análisis psicosocial y desarrollo de acciones comunitarias para el abordaje y prevención del suicidio (consumado e intentos) en personas jóvenes indígenas Bribri» ${ }^{2}$ (Arroyo y Herrera 2018), desarrollada como un proyecto de acción social de la Universidad de Costa Rica.

La investigación se realizó durante el período de agosto de 2015 a julio del 2017, con el objetivo principal de analizar el impacto psicosocial del suicidio en el tejido comunitario indígena Bribri.

\section{Investigación-acción-participativa como abordaje metodológico}

Para la realización del proceso de investigación, se asumió como modelo metodológico la investigación-acción-participativa (IAP), porque constituye un paradigma que reconoce el conocimiento popular, la pluralidad de saberes, la participación como ejercicio de poder y el carácter político y socialmente transformador del proceso de investigación (Montero 2006).

Se trabajó en las comunidades de Sepecue, Shiroles, Suretka y Bambú, pertenecientes al territorio indígena Bribri, ya que fueron las que presentaron mayor incidencia de intentos en el año 2014.

Para el abordaje comunitario del proceso de investigación, se realizó una aproximación a la problemática del suicidio en las personas jóvenes indígenas, considerando de manera intersistémica los siguientes niveles de análisis:

- Nivel social: se entendió como los marcos culturales e ideológicos. Para el proyecto de investigación refería al acercamiento a la Cosmovisión Indígena Bribri, donde fue de vital importancia la recuperación crítica de la historia de la comunidad. En este nivel se realizaron consultas con autoridades tradicionales como los awapas y kekepas ${ }^{3}$; así como a personas especializadas en la Cosmovisión indígena Bribri.

- Nivel comunitario: consistió en la aproximación a las formas de configuración del tejido social comunitario y sus particularidades. Para ello se realizaron consultas a organizaciones locales de

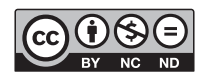


cada una de las comunidades de estudio y a organizaciones políticas. Así mismo, se realizaron consultas a nivel institucional, particularmente a personas representantes de los sectores sociales, de salud, seguridad, educación, justicia y protección, por ser los que tienen vinculación directa con la problemática de suicidio.

- Nivel relacional: se entendió como el nivel más cercano a las personas jóvenes; es decir: su familia, sus pares, su otro significativo. Se intentó el acercamiento a la vida cotidiana de las personas jóvenes, a sus patrones de actividad, roles, elementos simbólicos e identitarios, las relaciones interpersonales, entre otros (Arroyo y Herrera 2018, 12).

Es importante enfatizar que, al tratarse de una problemática tan sensible y compleja, el acercamiento inicial implicó un reto significativo, ya que el equipo de investigación era sikua (no indígena). Resultó clave en la investigación, el acompañamiento de dirigencia indígena y el ingreso al territorio, hasta contar con el permiso de las autoridades políticas y tradicionales.

La consulta se realizó en dos momentos: el primero abarcó desde agosto del 2015 a febrero del 2016, en el cual se realizó el levantamiento de información con actores institucionales. El segundo momento se realizó desde marzo a noviembre del 2016 y consistió en la consulta a actores comunitarios claves y persona jóvenes indígenas. En total, se realizaron 33 entrevistas, 9 conversatorios y 10 talleres comunitarios.

Es importante señalar que, inicialmente, el equipo de investigación se había planteado realizar entrevistas a profundidad con familias afectadas por suicidios. Sin embargo, el permiso para acceder a la privacidad de las familias no fue otorgado. Los resultados no presentan la mirada de las familias directamente afectadas. Sin embargo, en cada comunidad las participantes tenían vínculos con las familias sobrevivientes de suicidio.

Las entrevistas, conversatorios y talleres fueron sistematizados. La información derivada fue ordenada y agrupada por las categorías y niveles de abordaje comunitario para facilitar su análisis y la identificación de patrones y tendencias de las dimensiones de interés. Para garantizar la confidencialidad de las personas que participaron del proceso, se utilizaron referencias a los sectores que pertenecen en la presentación de los resultados.

Entre diciembre del 2016 y julio del 2017, se realizó el proceso de discusión de los hallazgos. Esta fue, quizás, la fase más intensa de la investigación porque, desde el abordaje metodológico y el posicionamiento ético del equipo, comprometido con el diálogo y el respeto, se dinamizaron diferentes espacios para la construcción colectiva de diálogos que problematicen los hallazgos, en un proceso de análisis-reflexión-acción: «a través del análisis, de la acción y reflexión colectivos, surge un proceso de concientización, que supone problematizar, a fin de que no se acepte como cotidiano, normal y lógico lo que es excluyente, insatisfactorio e injusto» (Montero 2006, 187).

\section{El suicidio desde un enfoque psicosocial}

Como concepto general, el suicidio se define como: «el acto consumado, intencional de finalizar con la propia vida. Incluye todas las muertes que son resultado directo o indirecto de comportamientos ejecutados por la propia víctima, quien es consciente de la meta que desea lograr» (Corpas 2011). Sin embargo, es necesario aclarar que la conceptualización del suicidio debe englobar el intento suicida como parte del fenómeno ya que, aunque no se concrete, dicho acto se dirige hacia el objetivo de quitarse la vida.

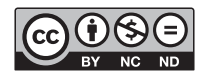


En la búsqueda de ampliar dicha conceptualización y el entendimiento de este fenómeno, Navas (2013) plantea que el suicidio es un fenómeno multicausal, entendido como resultado de la interacción de factores biológicos, genéticos, psicológicos, sociológicos, culturales y ambientales. Por su lado, Clemente y González (1996) realizan un recorrido por la conceptualización del suicidio a través de la historia, con el objetivo de abordarlo como un fenómeno social en el cual interactúan distintas variables que engloban la relación entre el individuo y su medio, concluyendo que «el suicidio es la expresión de la desmotivación del hombre ante la vida, desvinculándose de un medio social que le es frustrante y que no puede superar, pero que a su vez forma parte de sí mismo, de su identidad, representada por la dimensión social de toda existencia individual, y le es necesario, puesto que el hombre es ante todo no sólo un ser social, sino un producto de la sociedad en un primer momento, y de su interacción con la sociedad en un segundo momento» (Clemente y González 1996, 25).

Estas definiciones dirigen la mirada hacia la visión del suicidio como un fenómeno social, marcado por el contexto y las particularidades sociales, culturales y ambientales donde este ocurre. En este sentido, es importante profundizar en el "mecanismo de acción" del acto e intento suicida, ya que se debe entender como un proceso y no como un hecho en sí mismo. El suicidio y el intento suicida son la culminación de una serie de acciones que no ocurren aisladas unas de otras, ni desengranadas del ambiente inmediato de la persona que lo lleva a cabo.

Martínez (2017) profundiza en los elementos alrededor del acto suicida y su comportamiento, mirándolo como un proceso relacionado a una disposición autodestructiva, orientado a comunicar la intención de dar respuesta al conflicto que lo originó. Desde esta perspectiva, el autor cita el concepto de continuum autodestructivo de Adam (1985), buscando aclarar el acto suicida como un proceso progresivo. La persona autora señala que «el suicidio generalmente no es producto de una decisión espontánea, el continuum autodestructivo se va gestando en la intimidad del sufrimiento de una persona y se manifiesta a través de indicadores. Estos indicadores, junto con los factores de riesgo y las condiciones de potencial suicida, permiten predecir, prever y prevenir tanto los eventos en curso como nuevos acontecimientos autolesivos una vez ocurrido el primer intento» (Martínez 2017, 18-19).

La posibilidad de entender el acto o intento suicida como proceso, y diferenciar los elementos que intervienen a nivel individual y ambiental, plantea las herramientas para poder intervenir en dicha problemática. Martínez (2017) señala que hay que reconocer la existencia de un problema y un riesgo; y conocer cómo se conforman sus variables subjetivas, familiares y contextuales.

La persona autora afirma que la definición del suicidio implica un posicionamiento subjetivo, epistemológico, ideológico e institucional de quien lo define, ya que está determinado por coordenadas sociogeográficas y temporales. Es por esta razón, que el abordaje de este fenómeno debe tratarse con mucho cuidado, para que no se impongan nociones externas a la comunidad en que se trabaja (Martínez 2017). En este sentido, el acto suicida no se entiende como un pasaje para acabar con la vida, si no, un acto para terminar con el sufrimiento.

De esta manera, lo fundamental para entender el suicidio desde un enfoque psicosocial, es partir de que el suicidio no es una enfermedad, no es sinónimo de depresión; es un fenómeno multicausal y constituye un problema social, donde la persona materializa el sufrimiento de un sistema social (Castillo y Maroto 2017, Maroto 2016, Maroto 2017, Martínez 2008). Por tanto, sólo situando las coordenadas psicosociales que lo componen, se puede dar cuenta de este continuum autodestructivo.

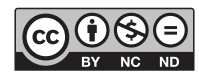

Rev. Reflexiones 98 (2) Julio-Diciembre, 2019: 7-22, E-ISSN: 1659-2859 


\section{El suicidio en territorios indígenas: una herida latinoamericana}

El suicidio es un fenómeno social y cultural que ha producido afectación en diferentes territorios indígenas latinoamericanos. Diferentes investigaciones (Corpas 2011; UNICEF 2012; Instituto Nacional de Medicina Legal y Ciencias Forenses 2015; Azuero et al. 2017) dan cuenta de que, el suicidio en poblaciones indígenas, se relaciona con violencias sociales, políticas, culturales y económicas.

Corpas (2011), realiza un análisis de 9 comunidades indígenas al norte y sur de América, donde el suicidio ocupa un lugar importante debido al número de intentos y consumaciones. De las comunidades estudiadas, las ubicadas al norte (Nuevo México, Alaska y Ontario), se caracterizan porque la mayor parte de suicidios se han dado, históricamente, en jóvenes entre los 11 y 34 años, asociando esta problemática al alto consumo de alcohol y a la dificultad de equilibrarse ante las demandas del desarrollo capitalista, al descubrirse como una minoría. En las comunidades ubicadas al sur de América, se destacan como factores de riesgo: las conductas de violencia dentro de las comunidades, la presión de multinacionales para apropiarse de las tierras, los cambios sociales, culturales y económicos.

El autor concluye que no es posible formar un perfil de las personas indígenas que se suicidan, a pesar de considerar la estadística que señala a los jóvenes entre 15 y 25 años como más vulnerables. Se considera que las razones son multicausales, aunque la irrupción de la occidentalidad, la pérdida de tierras y la frustración de las comunidades y sus miembros tienen un peso importante. El autor recalca que se debe tener claro que entre los factores socioculturales del suicidio están las drogas, el alcohol y la violencia; pero que hay algo anterior a estos factores que provocó su aparición.

En esta misma línea, el Instituto Nacional de Medicina Legal y Ciencias Forenses de Colombia (2015), corrobora algunas de las preocupaciones sobre la relación entre el suicidio y la población indígena. Realiza un análisis de los casos de suicidio en la población indígena colombiana, señalando que la población más afectada va de los 18 a los 24 años; pero también siendo significativa la cifra de suicidios en niños, niñas y adolescentes (23\% de los casos).

Dicho Boletín plantea que, en los 14 casos de niños, niñas o adolescentes, no hay un patrón específico, ya que las víctimas estudiaban, trabajaban en labores agrícolas o estaban desocupados (posibles labores domésticas). Y que, más de la mitad de los casos señalan como responsable del suicidio a aspectos sentimentales o afectivos. Así mismo, el análisis que realiza este Boletín, evidencia que, a pesar de las motivaciones personales, el suicidio es el resultado de dos tipos de violencia: la sociopolítica y la económica.

Por su parte, Azuero et al (2017) elaboran una revisión documental sobre el suicidio en poblaciones indígenas de América Latina. Los autores y las autoras realizaron una búsqueda sistemática en las bases de datos de PubMed, Scopus, PscycNET, Scielo y Google Scholar, que les permitió un resultado inicial de 1862 referencias, que fueron filtrados hasta la identificación de 21 artículos científicos con referencia al tema. Entre los hallazgos, llama la atención que los estudios vinculados al tema no se adscriben a los países con mayor población indígena como Bolivia, Guatemala o México. Los autores plantean que esta ausencia podría correlacionarse al hecho de la exclusión y marginalización de esta población, de la que se tiene poca información sobre casos de incidencia de suicidio o consumados.

La principal conclusión que se deriva, refiere a que los cambios en los estilos de vida generados por la industrialización, la degradación ambiental y la colonización, han incidido en que las poblaciones indígenas experimenten una «muerte cultural». Por otro lado, el apoyo social y familiar se identifican como los principales factores de protección ante esta problemática.

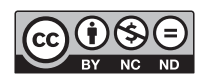


En los estudios revisados, los trastornos de salud mental en poblaciones indígenas no han sido profundizados como un factor incidente. Las personas autoras, asocian estas limitaciones a la relación entre la intervención medicalista, con sus nociones de salud y enfermedad de las poblaciones indígenas. Por otro lado, el alcoholismo es un problema importante que aparece como una manifestación de: la pérdida cultural e histórica, la discriminación étnica, la pobreza, el desempleo y la desintegración familiar.

Otro referente muy importante es el realizado por la UNICEF (2012), organización que presenta un informe sobre el abordaje de poblaciones indígenas de Perú, Brasil y Colombia; analizando las razones de la presencia de conductas suicidas. Este análisis se realizó a través de tres estudios de caso, una por país, tomando en cuenta los significados de las diferentes culturas, para así entender el lugar que tiene el suicidio en sus comunidades. Según lo observado, el suicidio se relaciona con el choque entre lo nuevo y lo viejo, entre la identidad asumida actualmente y las tradiciones de las comunidades:

\footnotetext{
Sin apoyo familiar, con amigos efímeros, sin saber cuál es su lugar, estos jóvenes continúan intentando sobrevivir en el aquí y el ahora. Cargan un trauma comunitario lleno de historias, contadas por sus parientes, de la explotación, de la violencia, de la falta de dignidad, en fin, la historia reciente de muchos pueblos indígenas. Historias cargadas de traumas, atadas a un presente de frustraciones e impotencia. En estas circunstancias estos jóvenes son el producto de lo que se suele llamar una Generación de Desorden de Estrés Post Traumático [...] Al no sentirse respetados, estos jóvenes quieren salir de la reserva, pero no cuentan siempre con el coraje suficiente para salir porque temen que tampoco tengan lugar en la ciudad (UNICEF 2012, 146).
}

Parece haber una carencia de herramientas o mecanismos de afrontamiento ante el choque cultural, dando como resultado, un trauma colectivo ante la dificultad de asimilación y entendimiento, debido a la violencia con la que los cambios llegan a las comunidades. El estudio aclara que no se debe entender el suicidio como la inserción de lo nuevo, cuya respuesta debe ser un abordaje que busque la recuperación del pasado como un fin en sí mismo; sino, asumir una posición ante la «hibridación» de las sociedades actuales, buscando construir formas en que las culturas se combinen, sin que sea violento para las nuevas generaciones.

Justamente el estudio cuantitativo realizado por el Instituto Nacional de Medicina Legal y Ciencias Forenses en Colombia muestra que «para los jóvenes (los más afectados) poder hablar y ser escuchados fue importante. La apreciación obtenida después de escuchar sus intervenciones, es que la difícil situación por la que atraviesan tiene que ver con los problemas que están viviendo, debido al deterioro ambiental de sus espacios de vida, que significa una disminución de sus recursos (madera, cacería, pesca), a la fuerte colonización de sus territorios y presencia de actores armados que han traído también violencia, desplazamiento, confinamiento, asesinatos, desarraigo y migración, situaciones que han conducido a una parálisis de la vida social y económica» $(2015,22)$.

La cita anterior resalta un aspecto fundamental a la hora de plantear una intervención, dejando claro que lo importante no sólo es conocer los factores de riesgo o protección de las y los adolescentes, sus problemáticas estructurales e individuales o los abordajes desde diversos paradigmas o disciplinas; sino el brindar una escucha a sus soluciones, sus deseos y posibilidades.

En esta línea, el estudio de la UNICEF (2012) plantea una serie de recomendaciones pertinentes de tener en cuenta para el abordaje en comunidades como las de Talamanca y en la escucha de las personas jóvenes, para poder facilitar soluciones desde su cosmogonía, deseos, intereses y en relación a sus posibilidades:

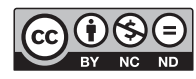


Cada cultura tiene sus propias categorías respecto a la salud y la enfermedad y cualquier medida a tomarse en relación con jóvenes y niños indígenas debe pasar necesariamente por un proceso de consulta previa, libre e informada. De hecho, tal vez la recomendación más importante es la de impulsar procesos que conduzcan a que la propia comunidad se apropie del problema, lo somete a reflexión y pruebe y establezca las estructuras y mecanismos necesarios para llevar a cabo una solución sostenible y duradera del problema. Cuanto mayor control tenga la comunidad, más certeros serán los análisis y menos riesgosas las decisiones (UNICEF 2012, 209).

\section{El suicidio en la Cosmovisión indígena Bribri}

Pérez define la cosmovisión indígena como «el conjunto de creencias, saberes y prácticas que se desarrollan de forma dinámica en una comunidad determinada, y que su sistema se fundamenta en relación con su entorno ecológico y cultural y, su memoria histórica-social» $(2014,7)$. En el caso de la Cosmovisión indígena Bribri, este conjunto de saberes crea la concepción del mundo: el Siwá, el cuerpo de conocimientos donde se resguarda la forma ancestral de entender el mundo y sus dimensiones. Para Bozzoli (1980), el Siwá es la historia, el sentido, el aire, la respiración, el viento, el alma, el conocimiento, el orden, el soplo de vida.

En la Cosmovisión Bribri, la realidad significa conexión entre todo lo existente, toda la naturaleza es sagrada y todo lo que hay en el mundo tiene vida y tiene un orden:

\footnotetext{
Un árbol, dentro de la cosmovisión indígena, es un hermano, un ser viviente, sólo si verdaderamente lo necesitamos, por razones muy justificadas para la sobrevivencia o porque así lo requieren las enseñanzas de Sibö y será necesario en la función de la curación, de un precepto ceremonial o ritual, respetamos su derecho a vivir, así mismo, con toda planta y todo animal y mineral. No consumimos la naturaleza por el mero hecho banal de lucro o de la estética mal entendida, no adquirimos una nueva silla porque la que teníamos pasó de moda. El blanco ve un árbol sin percatarse del ser viviente que este es, sino que pareciera que su percepción sólo está capacitada para traducir todo lo material en billetes, ve muchísimos billetes pegados al árbol. No se da cuenta de que es la casa de muchísimos seres vivientes, de que es un ser vivo que da frutos y se regenerará en el círculo de la naturaleza, tiene derecho a heredar futuras generaciones de su especie en este plantea, no percibe que las ramas son medicinales, que sus flores y frutos son fuente de vida de mariposas, pájaros y otros seres que tienen derechos al igual que el humano sobre este planeta (Morales 1969, referido por Salazar 1995, 157-158).
}

Por tanto, el valor de la vida se enfoca en todas las dimensiones, saberes y haceres. La vida se sostiene desde el principio del equilibrio, en el cual cada elemento de la vida es sagrado «en esta visión de mundo la relación ser humano-naturaleza se entiende como uno solo, es decir el ser humano es parte de la naturaleza y viceversa, ambos se encuentran en el mismo nivel, de manera que si se afecta a la naturaleza efectivamente existirán efectos sobre la persona misma» (Morales 2009, 12).

Al situar en estos principios de vida la problemática del suicidio, no es posible de significar como algo propio, ya que la Cosmovisión Indígena Bribri parte de la máxima que «si Sibö da la vida, sólo Sibö puede quitarla» (Especialista de Cosmovisión Bribi, comunicación personal) ${ }^{4}$.

El concepto de suicidio no existe como categoría lingüística, ni de vida, dentro de la cultura indígena Bribri. La noción de muerte implica el regreso al origen, al inframundo; pero cuando la muerte es inducida, el espíritu no puede hacer el regreso: «El tema de la muerte "normal" es muy importante. Cuando la muerte es natural, se regresa al inframundo, que es de donde se venía. Se cree que hay una vida después de la muerte; por eso hay celebración. Pero solo para las muertes naturales. Cuando la muerte es inducida, el espíritu se queda en la tierra sufriendo eternamente. Todo cuerpo de ser humano es de la naturaleza y el espíritu es de dios. El suicidio se entiende a partir de la inducción

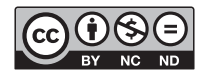

Rev. Reflexiones 98 (2) Julio-Diciembre, 2019: 7-22, E-ISSN: 1659-2859 
de personas de afuera que están enseñando eso, que se ve como "mal” (Especialista de Cosmovisión Bribi, comunicación personal).

En esta complejidad se intenta entender los factores incidentes de suicidio en el territorio indígena Bribri.

\section{La historia de resistencia Bribri}

Para situar las implicaciones psicosociales del suicidio en el territorio indígena Bribri, es importante contextualizar su historia de resistencia. Como se indicó anteriormente, las investigaciones en Latinoamérica sobre suicidio en poblaciones indígenas, establecen una relación directa con violencias sociales, políticas, económicas y culturales.

Al igual que otros pueblos indígenas, el territorio Bribri también está atravesado por una historia de violencia sistemática y despojos colonialistas. La aproximación a estos momentos históricos es fundamental para elaborar una lectura crítica y analítica de los factores incidentes del suicidio. En este sentido, Rojas (2009) propone la identificación de tres momentos históricos diferenciados que dan cuenta de esta resistencia que se juega en el enfrentamiento con la cultura occidental.

El primer momento lo sitúa de 1500 d.C., hasta la segunda mitad del siglo XIX, en el que se ubican los intentos de dominación español, a través de misiones y de la guardia colonial. Este período, que abarca casi tres siglos, representa para el pueblo indígena Bribri su primer momento de resistencia para salvaguardar su vida, su cultura, su política, su identidad y su Cosmovisión.

El segundo periodo es situado por el autor en la segunda mitad del siglo XIX, con la llegada de una nueva forma de opresión a través del capital. La intrusión de la compañía bananera United Fruit Company (UFCO), que planteó una nueva amenaza de dominación occidental; «en esta nueva modalidad en el enfrentamiento de la cultura tradicional bribri con la cultura occidental estaba mediada por la introducción de la tecnología: maquinaria, ferrocarriles, caminos de penetración y puentes lo que trajo consecuencias graves para los indígenas, como la imposición de una nueva forma de trabajo, la contratación "libre" de mano de obra y el "salario"» (Rojas 2009, 8).

El tercer periodo identificado por el autor, se ubica desde 1950 hasta la actualidad, el cual se da con la llegada del Estado y su imposición de ordenamiento social, legal y cultural. Este periodo «se caracteriza por instauración del sistema educativo formal, el fortalecimiento de la presencia de "nuevas religiones", la imposición de las políticas comunales, la presencia de la policía y el desarrollo de exploraciones petroleras en el área con serias implicaciones para las comunidades indígenas. Igualmente, se trata de definir la situación legal de estos territorios al declararlos reservas indígenas» (Rojas 2009, 9).

En estas violaciones se ponen de manifiesto la negación de la pertenencia al propio país ya que, para esta época, las personas indígenas no eran consideradas ciudadanas costarricenses. Es hasta 1991, producto de la movilización popular indígena, que se aprueba la Ley 7225 para otorgar ciudadanía a los pueblos originarios.

La presencia estatal en los territorios indígenas ha mantenido un corte asistencialista que, lejos de potenciar su autonomía, ha promovido: dependencias, debilitamiento de la fuerza organizativa y procesos de transculturación y aculturación. Sin embargo, en medio de este contexto, la reivindicación de las organizaciones indígenas sobre los derechos de los pueblos originarios, ha sido una de las principales vías de resistencia, respaldada en el Convenio 169 del 27 de junio de 1989.

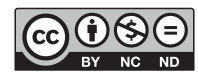


Como puede observarse, la historia del territorio indígena Bribri está atravesada por intentos sistemáticos de usurpación y colonización, que han derivado en un proceso de lucha y resistencia permanente.

Los procesos socio-históricos de exclusión, desigualdad y violencia, han zanjado una herida a nivel social que ha derivado en procesos de aculturación. Este término hace referencia a las transformaciones e impactos culturales surgidas cuando dos o más sistemas de tradiciones entran en contacto y choque (Smith 2002).

Cuando se habla de aculturación, se hace referencia a algo que se pierde o transforma, de un contacto donde una de las dos culturas tiene la posibilidad de ejercer mayor poder sobre la otra. De esta confrontación, pueden derivarse las siguientes estrategias de afrontamiento: «integración o biculturalismo, la cual representa fuertes vínculos con ambas culturas; asimilación que se refiere a la identificación exclusiva con la cultura dominante; separación la cual indica una fuerte identificación con el propio grupo y el rechazo al contacto con otros grupos, y marginalización, referida a la imposibilidad de establecer vínculos ni con el grupo de referencia, ni con la cultura dominante» (Smith 2002, 76).

Las consecuencias psicosociales de la aculturación dependen del nivel de contacto o confrontación. En el caso del territorio indígena Bribri, los procesos de violencia han sido profundos y sistemáticos y esto ha incidido en una cristalización social del sufrimiento. A este fenómeno se le denomina trauma psicosocial, porque refiere a un daño que no sólo se manifiesta en las personas, sino que está situada en la naturaleza de las relaciones sociales establecidas (Dobles 2016).

\section{El suicidio como la materialización de la muerte social y cultural previa}

El suicidio, desde un enfoque psicosocial, rompe con la patologización y se platea como un fenómeno social que apunta a una herida relacional. La conceptualización de Martín Baró (1990) de trauma psicosocial da herramientas para entender esta herida.

Etimológicamente trauma significa herida, por tanto, cuando se hace referencia a lo psicosocial, se entiende el trauma como una herida socialmente producida, que provoca daño en las subjetividades. Por tanto, en el trauma psicosocial: «(a) La herida ha sido producida socialmente, es decir, que sus raíces no se encuentran en el individuo, sino en la sociedad y (b) que su misma naturaleza se alimenta y se mantiene en la relación entre el individuo y la sociedad» (Martín Baró 1990, 78).

Para el autor se trata de la materialización en las personas del sufrimiento de las relaciones sociales, que se entiende como «un conjunto de síntomas psicosociales que se generan en una colectiva a partir de un proceso histórico caracterizado por intensa violencia política» (Simões 2014, 11).

En este sentido, desde la reflexión sobre esta herida en la centralidad del territorio y su impacto en el tejido social comunitario, se identifica una condición de impotencia en la capacidad de actuación. Esta se caracteriza, por un lado, por el reconocimiento de la angustia y el dolor que genera la muerte de las personas jóvenes; y por otro, el advenimiento de una especie de parálisis que imposibilita cualquier posibilidad de hacer: «Queremos hacer algo con los jóvenes y el doctor para ver qué está pasando, pero todavía no hemos hecho nada» (Líder indígena Bribi, comunicación personal).

A nivel comunitario, las percepciones sobre los factores incidentes de las personas representantes institucionales coinciden con las de las personas representantes de las organizaciones comunitarias:

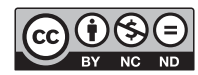


- Acceso tecnológico sin factores de protección: la entrada masiva de información y el acceso a realidades distintas, aporta modelos y formas de vida que genera tensión entre «lo propio y lo ajeno», «lo nuevo y lo viejo». El desconocimiento tecnológico por parte de los padres, dificulta las posibilidades de regular qué es lo que se mira: «Las tecnologías crean otras visiones y esquemas de vida. Absorben esas formas y pasan 24 horas viendo y oyendo cosas que los alejan de los valores de la vida espiritual» (Lideresa indígena Bribi, comunicación personal).

- Desintegración familiar, violencia doméstica y débil comunicación familiar: estas características lastiman las posibilidades de las personas jóvenes de sentirse acompañados: «En nuestra Cosmovisión todos somos responsables. Todos somos familia. Pero ahora las familias no son unidas. Antes si había una situación toda la familia se reunía. Ahora los hijos no respetan ni toman las decisiones de mamá y papá. Nosotros estamos acostumbrados a estar todos juntos, pero ahora hay mucho deterioro desintegración y deterioro de la base familiar» (Lideresa indígena Bribi, comunicación personal).

- Desvinculación de la cosmovisión indígena, del sentido comunitario y la vida espiritual, lo que incide en que «no se resiste a las presiones»: «Ahora Dios no es lo primero. Nosotros en nuestra Cosmovisión ponemos primero las cosas en manos de Dios. Desde que amanecemos hasta que anochecemos, agradecemos el viento, la lluvia, el sol. El alejamiento de los valores de la vida espiritual, hace que los jóvenes ya no resisten a las presiones» (Líder indígena Bribi, comunicación personal).

- Apropiación de ideas y valores que vienen de afuera, que crean rupturas con la propia Cosmovisión: «Antes era un honor ir a la montaña, escuchar a los Mayores. Ahora chocan las formas de ver las cosas» (Lideresa indígena Bribi, comunicación personal).

- Imposición de leyes sikuas que irrespetan los principios y las leyes indígenas Bribris: «Se ha irrespetado a nuestra forma de educar, nuestra forma de enseñar. Nos han impuestos leyes que no están hechas desde nuestra cultura. Una ley externa establece cuál es nuestro derecho. ¿Por qué debo respetar una ley que no me respeta? Mi ley no está escrita en papel, está escrita en el corazón» (Lideresa indígena Bribi, comunicación personal).

- El consumo de drogas como otra forma de muerte y autodestrucción: «Las drogas están en todo el territorio, no hay barrera, están en toda la comunidad. No hay proyecto, no hay espacios recreativos, no hay orientación de nada. Hay desorientación de la familia. Ante un problema leve, se piensa que todo se acaba» (Líder indígena Bribi, comunicación personal).

Por su parte, para las personas entrevistadas especialistas en Cosmovisión indígena Bribri (awapas y kekepas), los factores incidentes del suicidio se vinculan a:

- Imposición de la educación sikua desde una visión occidental, irrespetando las formas de conocimiento y cosmovisión Bribri.

- Proliferación de religiones que imponen su verdad y no respetan la cosmovisión Bribri.

- Imposición de formas culturales sikuas: aquello que viene de afuera y se impone sobre las tradiciones y las manifestaciones culturales propias, provocando rupturas y formas de alienación. La imposición de estas formas de colonización, atenta contra lo propio y desvincula a las personas jóvenes de su propia cultura y Cosmovisión.

- Leyes sikuas que no respetan la Cosmovisión Bribri: se impone un marco normativo desde una visión de derecho occidentalizada, que no integra las leyes tradicionales Bribri.

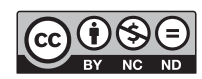

Rev. Reflexiones 98 (2) Julio-Diciembre, 2019: 7-22, E-ISSN: 1659-2859 
$\mathrm{Al}$ respecto, uno de los awa indígena Bribri sintetiza con suficiente claridad:

\begin{abstract}
Están sucediendo tres irrespetos. Primer irrespeto: Si irrespetamos las reglas, violentamos el equilibrio, nos hacemos daño. Segundo irrespeto: Sibö le dejó a cada semilla su par. Los pares están violentando las nuevas generaciones. Estamos como una chicha de pejibaye, asi de revueltos. Todos tenemos que respetar el mandato de Sibö. Él nunca dijo que llegaría el tiempo que cambiaran los pares. Él nos dejó dijo cómo teníamos que vivir, convivir, cuidarnos. Nos estamos comportando mal, no estamos cuidando los mandatos. Tercer irrespeto: Hay burlas, irrespetos, otra mentalidad. No se practican las tradiciones, no se baila el sorbone, no se hacen artesanías, no se construyen casas cómo se debe. Antes éramos colectivos, los hijos desde pequeños iban al campo a trabajar, ahora no saben. Los mayores dirigían la comunidad. Ya no se respetan. Este cambio se viene dando desde hace más de 20 años cuando llegó el Estado. Nosotros como pueblo Bribri teníamos nuestras reglas. El sistema educativo y la cultura sikua y sus leyes nos vienen violentando. Se rompe la unión de la familia, se nos arrebatan muchas cosas. Es la educación del olvido» (Awa Bribi, comunicación personal).
\end{abstract}

Para realizar un análisis de las impresiones de estos grupos es necesario recordar que, en la Cosmovisión Bribri, la realidad significa conexión entre todo lo existente: toda la naturaleza es sagrada y todo lo que hay en el mundo tiene vida y debe ser respetado. Desde esta noción de interconexión es que se entiende que la unidad Bribri es el «sujeto comunitario» (Morales 2009), lo individual no es una condición posible, se parte de la noción de que «todos somos familia», por tanto, la afectación de uno, es la afectación de todos.

Desde estos principios, el suicidio no es un acto posible y se vuelve impensable la muerte como decisión personal. Por tanto, las explicaciones en este nivel sobre porqué las personas jóvenes hacen un pasaje al acto de muerte, están asociadas a una imposición de una forma de ser sikua, que se explica con la expresión: «los Bribri no se suicidan, los sikuas sí» (Awa Bribi, comunicación personal).

En el caso de las personas jóvenes que participaron del proceso, estas asocian los factores incidentes a consumo de drogas, problemas familiares y desintegración familiar; debilidades de comunicación y comprensión por parte de padres y madres, problemas de pareja, falta de apoyos para afrontar problemas, falta de vida espiritual y falta de referentes de escucha.

Asumir estas percepciones como los únicos factores incidentes del suicidio, no permite un análisis crítico de sus dimensiones psicosociales. En el suicidio siempre hay algo anterior a estas manifestaciones, que provocaron su aparición y mantenimiento (Corpas 2011).

Para entender que es «lo anterior», es necesario hacer un análisis de la historia de resistencia indígena Bribri. Como se apuntó en apartados anteriores, Rojas (2009) establece tres períodos diferenciados en la historia indígena Bribri, en los cuales ha prevalecido la resistencia a los intentos de colonización y dominación, que se han realizado a través de la imposición de políticas y formas culturales occidentales a nivel social, cultural y educativo.

El análisis conduce a pensar que hay otra amenaza actual con otras manifestaciones, tensiones y rupturas: la globalización. Esta se entiende como un proceso de transformación económico, tecnológico, político y cultural; que implica relaciones de desigualdad y mecanismos de inclusión y de exclusión, de integración y de marginación (Giménez 2002).

Específicamente, y con respecto a las transformaciones culturales, Scholte (2000) plantea que la globalización remite a un proceso de desterritorialización; a «la proliferación de relaciones supraterritoriales, es decir, de flujos, redes y transacciones disociadas de toda lógica territorial, es decir, no sometidos a las constricciones propias de las distancias territoriales y de la localización en espacios delimitados por fronteras» (Sholte 2000 referido por Giménez 2002, 26).

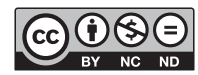

Rev. Reflexiones 98 (2) Julio-Diciembre, 2019: 7-22, E-ISSN: 1659-2859 
En este sentido, la ausencia de fronteras y barreras, así como la dinamización de lo global a través de mecanismos tecnológicos, crea procesos de tensión, ruptura y desterritorialización, que pueden devenir en formas de transculturación y aculturación.

Al situar estos procesos de transformaciones en el territorio indígena Bribri, la pregunta que surge es: ¿cómo se es una persona joven Bribri en esta época? Para intentar dar respuesta a esta interogante, se debe considerar que el ser Bribri está determinado por: la Cosmovisión, los principios y las leyes que rigen la vida, el conocimiento, el idioma, las tradiciones y las manifestaciones culturales. Si estas formas están siendo transformadas e, incluso, amenazadas por procesos de globalización, donde las fronteras se diluyen y las posibilidades de resistencia se trastocan o, en palabras de Zebadúa (2011), se cifran en un constante movimiento complejo entre el «afuera» y el «adentro»; es posible concluir que las personas jóvenes indígenas Bribris se encuentran en un lugar de vulnerabilidad caracterizado por dos tensiones:

- Una cultura externa, occidental colonialista, que intenta imponer modelos de vida sikua, que son excluyentes de lo identitario Bribri. Esto obliga a las personas jóvenes a tratar de acoplarse para no convertirse en «los otros», que son los excluidos.

- Una cultura indígena adultocéntrica entroncada, de cierta manera, en prácticas occidentales de relaciones asimétricas de hegemonía de los adultos sobre las personas jóvenes. Esto instala la percepción de que «los jóvenes están perdiendo su cultura», y se deposita en ellos la responsabilidad del olvido.

Este lugar de vulnerabilidad se profundiza con la falta de escucha, ya que la mayoría de las personas jóvenes entrevistadas afirmaron no sentirse escuchados por los adultos y no encontrar lugar para el reconocimiento de sus contradicciones. Entonces, si los jóvenes se encuentran en medio, jaloneados de un lugar a otro, entre el rechazo de afuera y el rechazo de adentro, entre la continuidad y la discontinuidad, nuestra pregunta debe ir dirigida a pensar qué posibilidades quedan para estas personas jóvenes.

Zebadúa (2011) retoma el concepto de linde para referirse a un «tercer espacio» difuso donde se encuentran dos espacios identitarios que están en vías de redefinirse; donde el conflicto puede estar dentro de uno de los territorios, en la delimitación de ambos o en un juego intersticial de fuerza entre los dos. Pero este tercer espacio plantea también un lugar de vacío, un «no lugar», donde también se juega la conexión y la desconexión con la posibilidad de ser y no ser: personas jóvenes desconectadas de su tradición, de su cultura, de su cosmovisión, de su idioma y de las personas adultas.

Se entiende desde este lugar de desconexión, que el suicidio es la materialización de la muerte social y cultural previa. Ya que cuando no hay conexión con lo identitario-cultural del ser Bribri y con los vínculos afectivos de las personas significativas, no hay posibilidad de relacionarse con la vida: «Porque si no sos Bribri, no sos nada» (Persona joven, comunicación personal).

A pesar del manifiesto debilitamiento cultural en la población indígena, producto de los procesos sistemáticos de violencia y aculturación, en el proceso de investigación se logró identificar en las personas jóvenes valores de orgullo con la pertenencia cultural: «La cultura Bribri es orgullo. Es mi forma de ser. Mi identidad. Estoy orgulloso de ser indígena y compartir costumbres. Somos responsables de no dejar que la cultura se pierda. No nos enseñaron a hablar Bribri. En la casa no se hablan, tal vez sólo los abuelos. Quizás no pensaron que se iba a perder. Cuando uno va a fuera y le preguntan si habla, da vergüenza. Es nuestro deber rescatar la lengua. Tenemos la responsabilidad de llevar la cultura y nunca olvidarla» (Persona joven, comunicación personal).

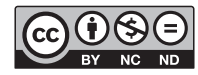


El reconocimiento de estas identificaciones en las personas jóvenes, son centrales para identificar fuerzas de resistencia, que posibilitarían la construcción de «un linde» o «tercer lugar», no desprovisto de la desconexión cultural y vincular, sino poblado de la construcción del sentido desde lo ancestral y lo identitario Bribri.

\section{Conclusiones}

El recorrido hecho por este estudio se suma a otras investigaciones que encuentran que el suicidio es la expresión de un trauma psicosocial, zanjado por prácticas sistemáticas de colonización que han incidido en el debilitamiento de las identidades culturales y de la construcción subjetiva indígena.

Los resultados de la investigación revelan que los factores incidentes del suicidio en el territorio indígena, están vinculados con este daño entendido como un trauma psicosocial asociado a procesos de intensa violencia política, social, cultural y económica. Encontramos que, en el caso del territorio indígena Bribri, el sufrimiento generado por estas prácticas violentas se ha transformado en autodestrucción $\mathrm{y}$ en el pasaje al acto suicida.

El suicidio es un fenómeno multicausal. En esta situación, el pasaje al acto no está directamente vinculado con terminar con la vida, sino con la finalización de un sufrimiento que resulta insoportable. Los factores incidentes en el territorio indígena Bribri, se asocian como rupturas de vínculos significativos (la familia, los pares, lo comunitario), exclusión, violencia y debilitamiento cultural donde, incluso la palabra «suicidio», es una nominación ajena e irruptora.

Sumado a esto, en las entrevistas realizadas, se puede determinar una desconexión del lazo social entre las personas adultas y las personas jóvenes. Esto ha actuado en detrimento de las posibilidades de apoyo para las personas jóvenes, quienes viven en tensión entre dos lugares que se presionan: un entorno globalizante ajeno a su territorio y un llamado a la cultura desde una demanda adultocéntrica.

Sin embargo, en medio de estas rupturas e inexistencias, también se concluye que las posibilidades de una nueva resistencia están situadas en recursos presentes en el territorio indígena Bribri; que exigen la construcción de puentes de escucha, diálogo y reencuentro cultural entre las personas mayores y las personas jóvenes indígenas Bribris:

- En el vínculo como factor de protección por excelencia y de conexión con la vida.

- En el fortalecimiento de lo cultural, del Siwá de los Pueblos Bribris, como vía de resistencia.

Con base en estos dos hallazgos, se sugiere realizar abordajes comunitarios desde una praxis política, basada en la construcción de relaciones humanizantes que despierten lo humano: «Considero que lo más profundo de nuestra realidad humana estriba precisamente en hacernos mutuamente, construirnos mutuamente» (Martín- Baró 1989, citado por Dobles 2016, 201).

La prevención del suicidio se basa en la previsión (Martínez 2008). La noción de que en el vínculo y el fortalecimiento cultural se encuentran los factores de protección por excelencia, permite clarificar que la ruta de un abordaje preventivo, desde un modelo de salud mental comunitario, conlleva a la construcción de prácticas comunitarias humanizadoras que permitan «el encuentro, la autoestima, la integración y las posibilidades reales de construir subjetividad e intersubjetividad» (González 2010, referido por Dobles 2016, 202).

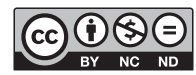


Esta ruta sólo será posible si estos hallazgos reivindican los saberes ancestrales y posibilitan la construcción colectiva de otros nuevos que construyan, entre ese «adentro» y ese «afuera», una posibilidad de resistencia como un lugar propio, que posibilita la re-existencia.

\section{Notas}

1. Esta investigación es el resultado de un proceso de reflexión y construcción conjunta entre líderes y lideresas indígenas, personas jóvenes indígenas y personas funcionarias de instituciones del cantón de Talamanca, que fue realizado entre agosto del 2015 y julio del 2017. Este equipo de investigación sólo es un vocero, el saber escrito en estas páginas, le pertenece a ellos y ellas.

2. Esta investigación contó con el apoyo del equipo de asistentes: Bach. Mariana Alpízar Guerrero, Bach. Ana María Jiménez Hidalgo y Bach. Eugenia Murillo Fonseca (mencionados por orden alfabético según su apellido).

3. Los awapas son los médicos y sabios indígenas Bribris, en tanto que los y las kekepas son las personas Mayores, no por edad, sino por sabiduría.

4. Todas las entrevistas fueron confidenciales y los nombres de los entrevistados se han ocultado por mutuo acuerdo. Para la referencia, se utiliza el cargo.

\section{Referencias}

Arroyo Helga, y Damián Herrera. 2018. «Diagnóstico comunitario: análisis psicosocial del suicidio en personas jóvenes indígenas Bribri». Manuscrito inédito. Archivo de Microsoft Word.

Martin-Baró, Ignacio. 1990. Psicología Social de la Guerra: trauma y terapia. San Salvador: UCA Editores.

Azuero, Andrés et al. 2017. «Suicide in the Indigenous Population of Latin America: A Systematic Review». Rev Colomb Psiquiat, 46 (octubre-diciembre): 237-242. http://dx.doi.org/10.1016/j. rcp.2016.12.002.

Instituto Nacional de Medicina Legal y Ciencias Forenses. 2015. Boletín Epidemiológico. Suicidio de Indígenas en Colombia 2010-2014. Grupo Centro de Referencia Nacional sobre Violencia. Colombia. http://www.medicinalegal.gov.co/documents/20143/57952/Suicidio+de+Ind\%C3\%AD genas+en+colombia.+2010-2014.pdf

Bozzoli, María Eugenia. 1980. El nacimiento y la muerte entre los Bribri. San José: Editorial Universidad de Costa Rica.

Castillo, Carolina, y Adriana Maroto. 2017. «El suicidio desde un enfoque psicosocial y de salud comunitaria: Los resultados del diagnóstico de Santa María de Dota, San José, Costa Rica». Anuarios de estudios centroamericanos 43: 447-472. DOI: 10.15517/AECA.V1I1.28856

Clemente, Miguel, y Andrés González. 1996. Suicidio. Una alternativa social. Madrid: Editorial Biblioteca nueva.

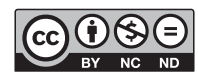


Corpas, José. 2011. «Aproximación social y cultural al fenómeno del suicidio. Comunidades étnicas amerindias». Gazeta de Antropología 27 (2), artículo 33: 1-15. http://hdl.handle.net/10481/18682

CCSS (Caja Costarricense de Seguro Social). 2014. Departamento de Epidemiología. «Base de Datos 2014». Área de Salud Talamanca. Talamanca.

Dobles, Ignacio. 2016. Ignacio Martín-Baró: Una lectura en tiempos de quiebres y esperanzas. San José: Editorial Arlekín.

Giménez, Gilberto. 2002. «Globalización y cultura». Revista Estudios Sociológicos 20 (58): 23-46. https://www.redalyc.org/articulo.oa?id=59805802

INEC (Instituto Nacional de Estadística y Censos). 2011. «Censo Nacional de Población». San José: INEC.

Maroto, Adriana. 2016. «Revisión de la investigación sobre suicidio en Costa Rica (1998-2013): Principales hallazgos». Revista de Ciencias Sociales 154: 149-158. DOI 10.15517/RCS.V0I154.29199

2017. «El suicidio en el ámbito comunitario: lineamientos para su abordaje». Reflexiones 96: 27-39. DOI 10.15517/RR.V96I1.30630

Martínez, Carlos. 2008. «El abordaje comunitario de la crisis suicida en sus diferentes ámbitos de intervención». Manuscrito inédito. Archivo de Microsoft Word.

2017. Suicidología Comunitaria para América Latina. Buenos Aires: Alfepsi Editorial.

Montero, Maritza 2006. Hacer para transformar: el método en la psicología comunitaria. Buenos Aires: Editorial Paidós.

Morales Pita, Maycol Alonso. 2009. «Siwa Ka: Informe de Final». Manuscrito inédito. Archivo de Microsoft Word.

Navas, Wendy. (2013) «Abordaje del Suicidio en Atención Primaria». Revista Médica de Costa Rica y Centroamérica 70 (abril-junio): 219-225. http://www.binasss.sa.cr/revistas/rmcc/606/art4.pdf

OIJ (Organismo de Investigación Judicial). 2014. «Personas fallecidas en Costa Rica por suicidio en el 2014». https://www.poder-judicial.go.cr/planificacion/index.php/ estadisticas-2014?download=444:suicidios-analisis

Pérez, Marcela. 2014. «Cosmovisión indígena y oralidad». Manuscrito inédito. Archivo de Microsoft Word.

Rojas, Daniel. 2009. Dilema e identidad del pueblo Bribri. San José: Editorial UCR.

Salazar Palavicini, Maritza. 1995. «El paradigma de las tradiciones indígenas Bribri de Costa Rica y la Psicología». Tesis de maestría, Universidad de Costa Rica.

Scholte, Jan. 2000. Globalization. Nueva York: St. Martin's Press.

Simões, Bruno. 2014. «Parecer técnico Psicológico sobre los impactos psicosociales de la población indígena Krenak». Manuscrito inédito. Archivo de Microsoft Word.

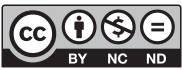

Rev. Reflexiones 98 (2) Julio-Diciembre, 2019: 7-22, E-ISSN: 1659-2859 
Smith Castro, Vanessa. 2002. «Aportes a la comprensión de la identidad étnica en niños y niñas de grupos étnicos minoritarios». Revista de Ciencias Sociales 98: 71-82. https://www.revistacienciassociales.ucr.ac.cr/images/revistas/RCS96/05.pdf

UNICEF. 2012. Suicidio adolescente en pueblos indígenas: tres estudios de caso. Panamá: Ed. UNICEF.https://www.unicef.org/peru/spanish/SuicidioAdolescentePueblosIndigenas(1).pdf

Zebadúa, Juan Pablo. 2011. «Cultura, identidades y transculturalidad. Apuntes sobre la construcción identitaria de las juventudes indígenas». Revista LiminaR. Estudios sociales y humanísticos 9: 36-47. http://liminar.cesmeca.mx/index.php/r1/article/view/60 